\title{
Numerical study of the scavenging process in a large two-stroke marine engine using
} URANS and LES turbulence models

Nemati, Arash; Ong, Jiun Cai; Jensen, Michael Vincent; Pang, Kar Mun; Mayer, Stefan; Walther, Jens Honore

\section{Published in:}

S A E Technical Papers

Link to article, DOI:

$10.4271 / 2020-01-2012$

Publication date:

2020

Document Version

Peer reviewed version

Link back to DTU Orbit

Citation (APA):

Nemati, A., Ong, J. C., Jensen, M. V., Pang, K. M., Mayer, S., \& Walther, J. H. (2020). Numerical study of the scavenging process in a large two-stroke marine engine using URANS and LES turbulence models. $S A E$ Technical Papers, [2020-01-2012]. https://doi.org/10.4271/2020-01-2012

\section{General rights}

Copyright and moral rights for the publications made accessible in the public portal are retained by the authors and/or other copyright owners and it is a condition of accessing publications that users recognise and abide by the legal requirements associated with these rights.

- Users may download and print one copy of any publication from the public portal for the purpose of private study or research.

- You may not further distribute the material or use it for any profit-making activity or commercial gain

- You may freely distribute the URL identifying the publication in the public portal 


\title{
Numerical study of the scavenging process in a large two-stroke marine engine using URANS and LES turbulence models
}

\author{
Arash Nemati ${ }^{\mathrm{a}}$, Jiun Cai Ong ${ }^{\mathrm{a}}$, Michael Vincent Jensen ${ }^{\mathrm{a}}$, Kar Mun Pang ${ }^{\mathrm{b}}$, Stefan Mayer ${ }^{\mathrm{b}}$ and \\ Jens Honoré Walther ${ }^{\mathrm{a}, \mathrm{c}}$ \\ aDepartment of Mechanical Engineering, Technical University of Denmark, 2800 Kgs. Lyngby, Denmark \\ b MAN Energy Solutions, Teglholmsgade 41, 2450 Copenhagen SV, Denmark \\ ${ }^{c}$ Computational Science and Engineering Laboratory, ETH Zürich, $\mathrm{CH}-8092$ Zürich, Switzerland
}

\section{ABSTRACT}

A computational fluid dynamics study of the scavenging process in a large two-stroke marine engine is presented in this work. Scavenging which is one of the key processes in the two-stroke marine engines, has a direct effect on fuel economy and emissions. This process is responsible for fresh air delivery, removing the combustion products from the cylinder, cooling the combustion chamber surfaces and providing a swirling flow for better air-fuel mixing. Therefore, having a better understanding of this process and the associated flow pattern is crucial. This is not achievable solely by experimental tests for large engines during engine operation due to the difficulties of measuring the flow field inside the cylinder. In this study, the axial and tangential velocities are compared and validated with the experimental results obtained from Particle Image Velocimetry (PIV) tests [1]. The simulations are conducted using both Unsteady Reynolds Averaged Navier Stokes (URANS) and Large Eddy Simulation (LES) turbulence models. We observe in general, there is a good agreement between the numerical and experimental results. The flow inside the cylinder is studied in different locations related to the bottom of the scavenging ports during the period with open exhaust valve. Moreover, the replacement of combustion products with fresh scavenge air is analysed. The effective flow angle is calculated for the air flow through the scavenging ports. It is found that the effective flow angle is different from the geometrical angle of the ports $\left(20^{\circ}\right)$. Results illustrate better performance of LES, especially in the prediction of the tangential velocity which is crucial for the simulation of an accurate swirl and air-fuel mixing inside the marine engines. LES predicts a uniform profile for the tangential velocity at the top of cylinder which is consistent with the experimental results while URANS predicts a solid body rotation.

\section{INTRODUCTION}

Large two-stroke marine diesel engines dominate as prime movers for cargo ships due to their high efficiency, low fuel consumption, and reliability [2]. The scavenging process delivers fresh air to the combustion chamber, removes combustion gases in the cylinder, cools the combustion chamber surfaces, and distributes fuel in the cylinder [3], playing an important role in the two-stroke marine engines. Therefore, the efficiency of the scavenging process is crucial for the total engine performance. Uniflow scavenging, which is widely used in large two-stroke engines, is able to remove exhaust gas much better than loop scavenging and cross scavenging as well as offering a higher scavenging efficiency [4]. It also provides more control on the flow by optimizing the exhaust valve opening and closing timing. Another reason for using the uniflow scavenging system in marine engines is the high ratio of the stroke to the cylinder diameter (S/D).

Experimental studies on the scavenging flow inside the full-size firing marine diesel engines are limited due to difficulties of flow measurement inside the large cylinders of these engines during combustion [1,5]. The experimental tests are mostly limited to the steady-flow scavenging process $[6,7,8]$ or dynamic-flow conditions [4, 9] on small model engines which differ significantly compared to those in the full-size engine. Therefore, computational fluid dynamics (CFD) studies are essential to obtain an understanding of the scavenging flow inside the large engine cylinder. There are several CFD studies of the scavenging flow in a model engine using Unsteady Reynolds Averaged Navier Stokes (URANS) and Large Eddy Simulation (LES) models [3, 10, 11, 12, 13]. However, three-dimensional (3D) CFD simulations on the large two-stroke engines are limited. Lamas and Vidal [14] performed a CFD analysis of the scavenging process 
in a MAN B\&W 7S50MC two-stroke marine diesel engine using the URANS model. They validated the in-cylinder pressure with experimental data during the scavenging process and proposed their CFD model as a useful tool for designing the scavenging system of new large twostroke engines. Andersen et al. [15] used URANS to simulate the scavenging process for different loads in the large two-stroke MAN 4T50ME-X test engine. They studied important scavenging parameters such as scavenging and charging efficiencies and bulk purity of the air inside the cylinder. They concluded that the scavenging efficiency and bulk purity of the air decreases with increasing engine load. Cao et al. [16] simulated a full engine cycle for the MAN Diesel \& Turbo 6S35ME-B9 engine under firing condition including the scavenging process. They validated the pressure curve for the cycle and studied the effect of ports angle and exhaust valve closing (EVC) timing on fuel consumption and nitrogen oxides $\left(\mathrm{NO}_{\mathrm{x}}\right)$ emission. They concluded that $20^{\circ}$ port angle has the lowest fuel consumption and $\mathrm{NO}_{\mathrm{x}}$ emission in comparison with $10^{\circ}$ and $30^{\circ}$ port angles. Their results clarified that retarding the EVC timing leads to a lower scavenging and maximum pressure which consequently increases the brake specific fuel consumption (BSFC) and decreases the $\mathrm{NO}_{x}$ emission. Foteinos et al. [17] proposed a three-zone model for the scavenging process in a large two-stroke engine using the results from CFD simulations. They used the URANS model for CFD simulation and validated their results with Particle Image Velocimetry (PIV) measurements of a model engine [18]. They studied the effects of different engine loads and inlet port angles on the scavenging flow. They concluded that the mixing of air and burned gas increases with engine load.

To the authors' best knowledge, a comparison between the performance of the URANS and LES in simulating the scavenging flow in a full-size engine has yet to be performed. Due to the importance of the scavenging process on the air-fuel mixing and combustion, the accuracy of the flow simulation in these engines is crucial. Set against this background, the present study investigates the scavenging flow in a full-size large two-stroke marine engine using the URANS and LES turbulence models. The in-cylinder flow is first validated with experimental data. The axial and tangential velocity snapshots are compared for the URANS and LES at different crank angle degrees (CADs). In addition to these, the radial profiles of axial and tangential velocities are presented and compared for the URANS and LES. Besides that, the effective flow angle is calculated for the air flow through the scavenging ports and the replacement of burned gas with fresh air is studied and compared for the URANS and LES models.

\section{NUMERICAL MODELING}

\section{Engine specifications}

The current CFD models are validated using the experimental data provided by MAN Energy Solutions in Copenhagen. The pressure and PIV measurements were car- ried out on the 4T50ME-X research engine [1]. Details of the engine specifications are presented in Table 1. As mentioned in Table 1, the simulation is carried out for $25 \%$ engine load. The PIV tests were only conducted at $25 \%$ load due to problem with deposits from combustion products and lubrication at higher engine loads [1]. At $25 \%$ engine load, auxiliary blowers were used to increase the pressure in the scavenge receiver as the turbo charger is unable to generate a sufficient pressure drop across the engine. The simulations only consider one of the engine cylinders and initiate from exhaust valve opening (EVO).

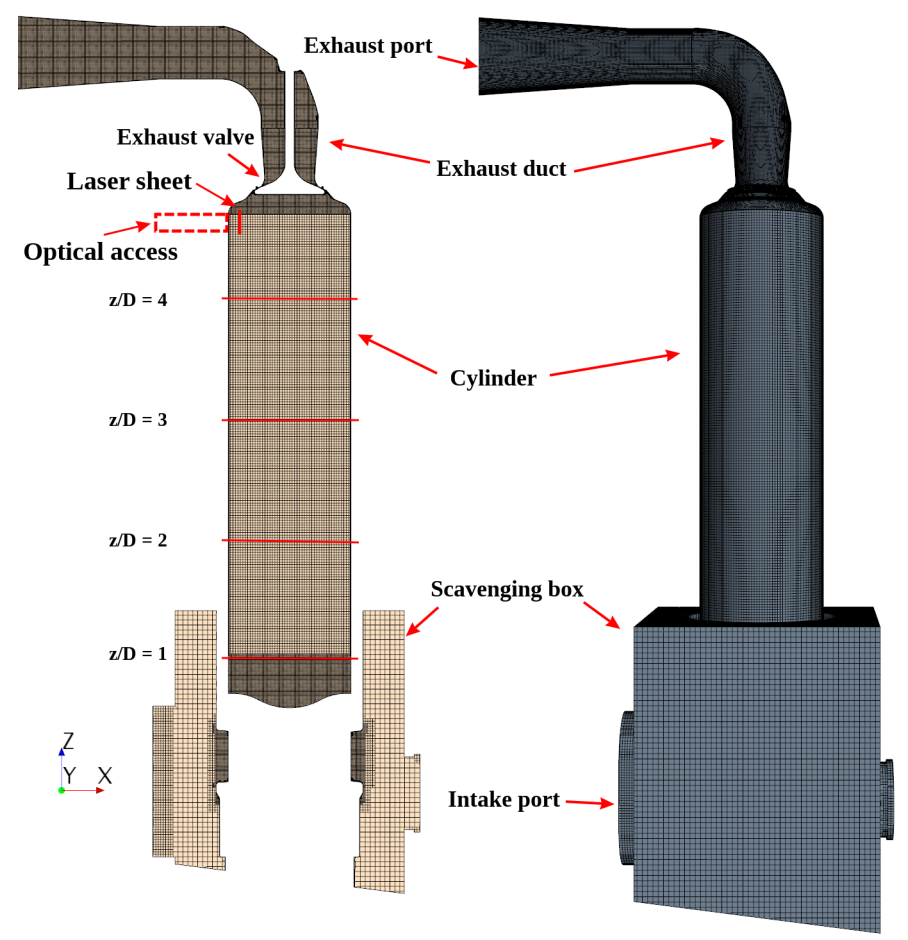

Figure 1: Overview of mesh at EVO - red line probes located at 4 different locations related to the bottom of the scavenging ports location of laser sheet and optical access for PIV measurements.

Table 1: 4T50ME-X engine specifications and operating conditions used in the current simulations.

\begin{tabular}{|l|l|}
\hline Engine type & Two-stroke, diesel \\
\hline Bore & $500 \mathrm{~mm}$ \\
\hline Stroke & $2200 \mathrm{~mm}$ \\
\hline Connecting rod & $2885 \mathrm{~mm}$ \\
\hline Exhaust valve opening (EVO) & $128 \mathrm{CAD}$ ATDC \\
\hline Exhaust valve closing (EVC) & $257 \mathrm{CAD}$ ATDC \\
\hline Ports per cylinder & 30 \\
\hline Engine speed & $77.5 \mathrm{rpm}$ \\
\hline Engine load & $25 \%$ \\
\hline
\end{tabular}

The scavenging process in this engine can be divided into three main phases. The blow-down phase which starts at EVO and ends at inlet port opening (IPO). The forcedscavenging phase which is from IPO to inlet port closing 
(IPC). Finally, the push-out phase that starts at IPC and ends at EVC. An overview of the computational domain is presented in Figure 1 which consists of a scavenging box, cylinder, exhaust valve, and exhaust duct.

\section{Numerical simulation}

A 3-D CFD simulation is performed using the commercial CFD software STARCCM+ version 14.06.012-R8. The turbulent flow is modeled using the URANS method with the Realizable $k-\epsilon$ model [19] and the LES model using the wall-adaptive local eddy-viscosity (WALE) subgridscale model. The temporal terms in the URANS equations are discretized using a first-order scheme, while the second-order schemes are used in the LES model. The mesh size is different in various parts of the domain. Inside the cylinder the mesh size is $0.01 \times D$ ( $D$ is the cylinder diameter) and around the scavenging ports it is $0.005 \times \mathrm{D}$. Furthermore, due to importance of valve opening and closing, the mesh size is $0.002 \times \mathrm{D}$ in the exhaust valve gap. The time step size is set to 0.0023 CAD such that the mean Courant number for the cylinder remains below 0.07 . The momentum, mass, and energy equations in both the URANS and LES cases are spatially discretized with a second-order scheme. The initial species in the scavenging box is considered to be air (a mixture of $\mathrm{N}_{2}$ and $\mathrm{O}_{2}$ ) and the initial species in the cylinder and exhaust duct are considered to be combustion products i.e. a mixture of $\mathrm{N}_{2}, \mathrm{O}_{2}, \mathrm{H}_{2} \mathrm{O}$ and $\mathrm{CO}_{2}$ (assuming the air-fuel equivalence ratio of $\lambda=2.1$ ). The pressure boundary condition on the scavenging box inlet is a time-dependent value obtained from experimental data which is depicted in Figure 2 and the outlet pressure boundary condition is a time-averaged value $\left(P_{\text {outlet }}=1.4 \mathrm{bar}\right)$. A no-slip condition is applied to all wall boundaries. The initial tangential velocity inside the cylinder is computed based on a second-order polynomial equation. This equation is obtained based on the experimental values of the tangential velocity in the position of the laser sheets at $98 \mathrm{~mm}$ and $195 \mathrm{~mm}$ from the cylinder axis (please see [1]). The initial axial velocity is set using a linear velocity profile, starting from zero in the vicinity of the exhaust valve and increasing to be the same as the piston velocity near the piston.

\section{RESULTS AND DISCUSSION}

For validation of the simulation, the in-cylinder pressure during the scavenging process is compared with the experimental data. In addition, the PIV results measured by Hult et al. [1] for the axial and tangential velocity components at the position of $195 \mathrm{~mm}$ from the cylinder axis are also utilized in the validation. The comparison of the predicted in-cylinder pressure during the scavenging process from the CFD simulation with experimental data is presented in Figure 3. From the figure, the CFD simulations are able to capture the frequencies in the cylinder pressure. It is also worth mentioning that there is negligible difference between the URANS and LES in the prediction of the in-cylinder pressure during the scavenging process. Therefore, pressure validation alone is not sufficient to en-

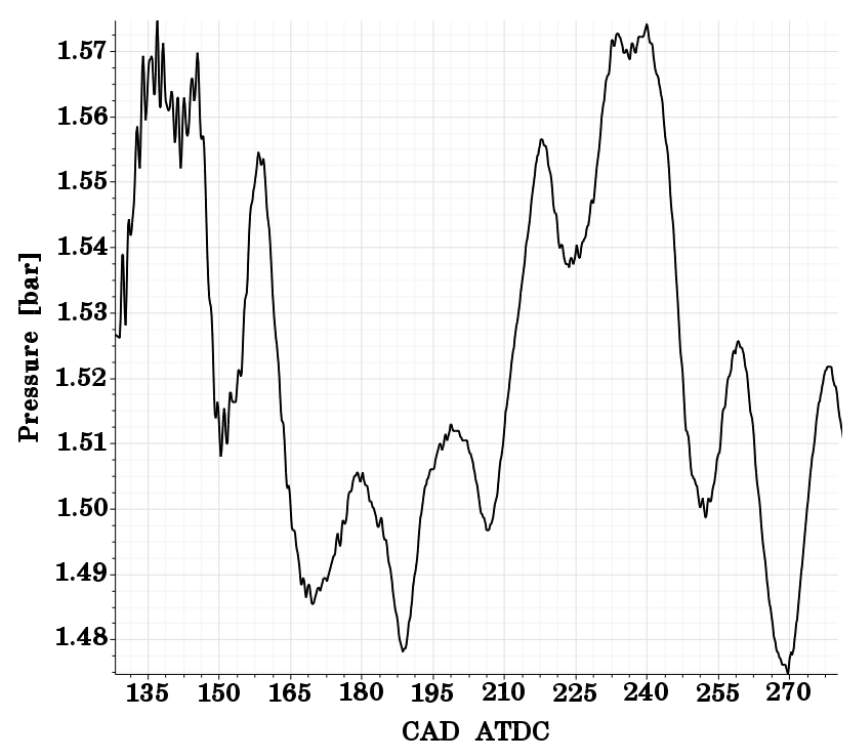

Figure 2: Scavenging box inlet pressure boundary condition

sure that the simulated flow inside the cylinder is correctly captured and to compare the model performance between the URANS and LES modeling approaches. Next, the velocity flow field predicted by the current CFD models is validated using the experimental data as shown in Figure 4 . The axial and tangential velocity components are calculated by averaging on a surface with the same size as the experimental laser sheet $(50 \times 50 \mathrm{~mm})$ located in a distance of $195 \mathrm{~mm}$ from the axis of the cylinder. The location of the flat laser sheet is marked in Figure 1 which the top position of the laser sheet coincides with the bottom of the valve when fully open.

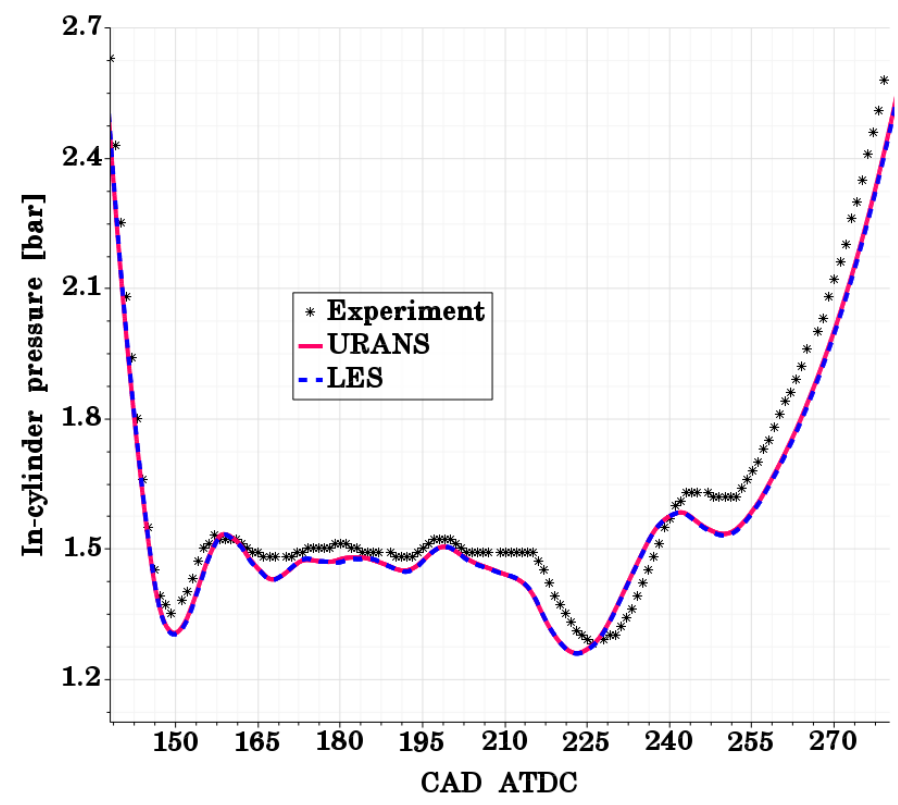

Figure 3: Comparison of in-cylinder pressure during the scavenging process with experimental data

From the figures, the CFD simulations are able to predict 
the evolution of the axial and tangential velocities which corresponds with the experimental data reasonably well.

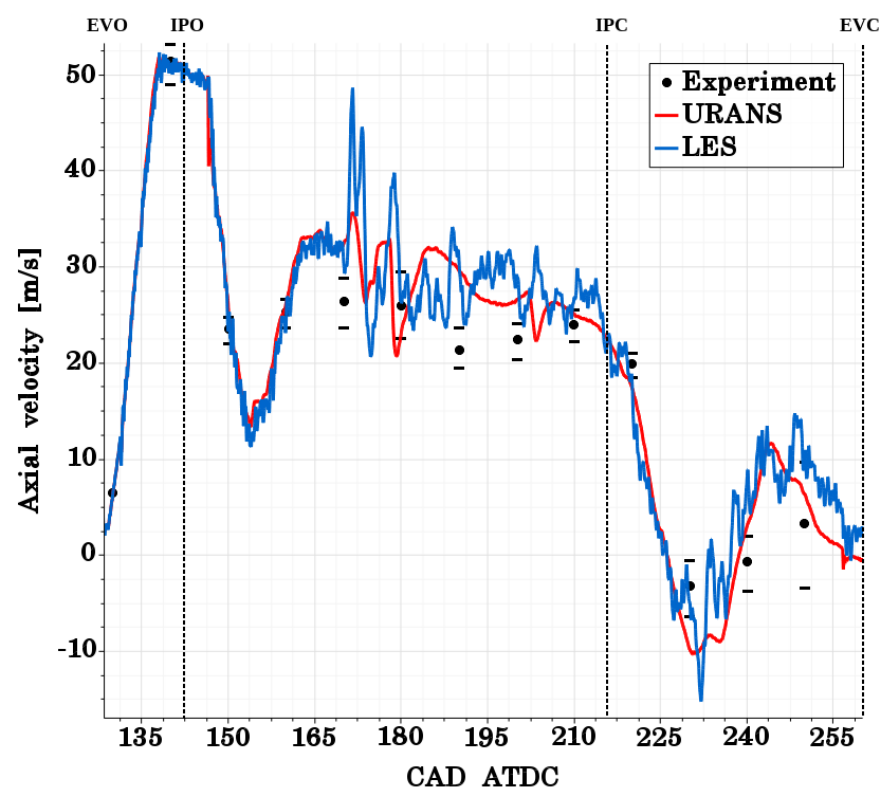

(a)

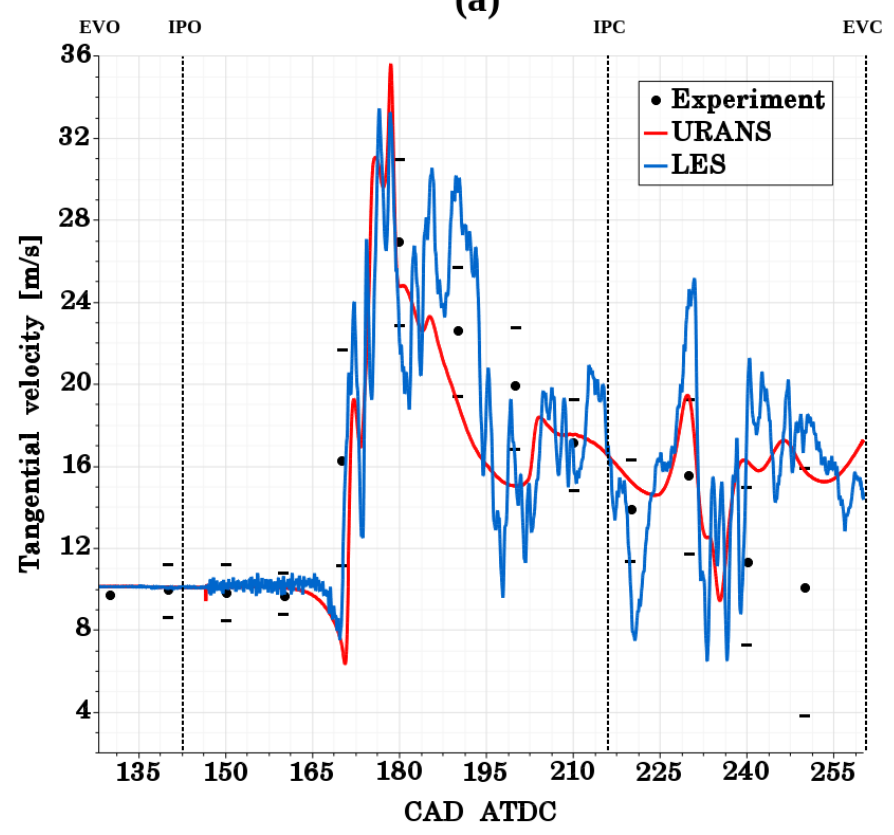

(b)

Figure 4: Comparison of (a) axial velocity (b) tangential velocity obtained from URANS and LES models with experimental data

When the exhaust valve opens at 128 CAD after top dead center (ATDC), the axial velocity in the laser sheet position, suddenly increases to around $50 \mathrm{~m} / \mathrm{s}$ due to a high flow rate during the blow-down phase cf. Figure 4a. Then, the axial velocity remains almost constant for around 10 CADs. After uncovering the ports at IPO, the pressure difference across the cylinder reduces resulting in a significant decrease in axial velocity to around $15 \mathrm{~m} / \mathrm{s}$. As the scavenging ports continue to open, the axial velocity increases slightly and remains almost constant due to an approximately constant pressure difference between the scavenging box inlet and exhaust port outlet.

During this phase, the driving force of the flow is the pressure difference between the scavenging receiver and the exhaust receiver. It can be seen in Figure 4a that LES and URANS predict similar axial velocities from EVO (around 128 CAD ATDC) to 170 CAD ATDC. This is likely due to that the axial velocity in the laser sheet position is only affected by outgoing flow through the valve. However, at around 170 CAD ATDC, the incoming air through the scavenging ports reaches the position of the laser sheet and LES starts to predict a different axial temporal profile than URANS. At around 217 CAD ATDC, when the scavenging ports are completely closed, the forced scavenging phase ends and the axial velocity drops down to low values due to the absence of a driving pressure difference between the inlet and the outlet which moves the flow through the cylinder. The negative axial velocity indicates a small backflow of exhaust gas at these CADs. After IPC, the push-out phase starts where the piston motion pushes the in-cylinder gas out through the exhaust valve. Due to the reduction of flow area in the valve closing time, the axial velocity increases again at around 237 CAD ATDC and then decreases due to the complete closing of the valve.

The temporal variation of the tangential velocity is presented in Figure $4 \mathrm{~b}$ for the URANS and LES models. It can be seen that the opening of the exhaust valve does not have a significant effect on the tangential velocity, as such this velocity component remains almost constant until 170 CAD ATDC. This observation implies that the exhaust valve opening have insignificant effect on the swirling flow inside the cylinder. Moreover, it is clear that the tangential velocity does not respond immediately to the opening of the scavenging ports. This is because the fresh scavenge air needs time to reach the laser sheet area which is located near the head of the cylinder. It takes around $25 \mathrm{CAD}$ for the swirling flow to travel inside the cylinder between the scavenging ports and the laser sheet position [1]. The CFD simulations are able to predict the tangential velocity rising time reasonably well. After an intense increase, the tangential velocity decays which is also predicted by the CFD simulation. The increase in the tangential velocity at 225 CAD ATDC is due to the backflow of exhaust gas (which is also obvious in the axial velocity profile) which immediately increases the tangential velocity on the laser sheet located below the valve inside the cylinder (please see [1] for the laser sheet position).

To visualize the flow inside the cylinder, the streamlines at 150 CAD ATDC for the URANS turbulence model are presented in Figure 5. The red and blue lines are the streamlines with seeding source at two scavenging ports in the left and right hand side of the scavenging box. These streamlines move inward along almost straight lines and then wind around the vortex core. The flow separation above the ports creates a vortex ring, shown by the black streamlines seeded inside the cylinder. 
After validating the velocity components, the spatial distribution of the axial and tangential velocity fields in the cross-section of the engine for different CADs ATDC are presented in Figures 6 and 7 for the URANS and LES models. As it can be seen in Figure 6, at 150 CAD ATDC which is around 6 CADs after IPO, the incoming air from different scavenging ports meets in the center of the cylinder which leads to a loss of the kinetic energy. This is due to the higher radial flow through the ports in the opening time of the scavenging ports (this will be discussed in detail in the next parts of the paper about effective flow angle). At 150 and 160 CADs ATDC, there are three main areas inside the cylinder with negative axial velocities: the bottom of the cylinder (region 1), the sides near the cylinder wall (region 2) and the center of the cylinder (region 3). These regions are illustrated in Figure 6a for $150 \mathrm{CAD}$ ATDC. These regions with negative axial velocity are the re-circulation areas.

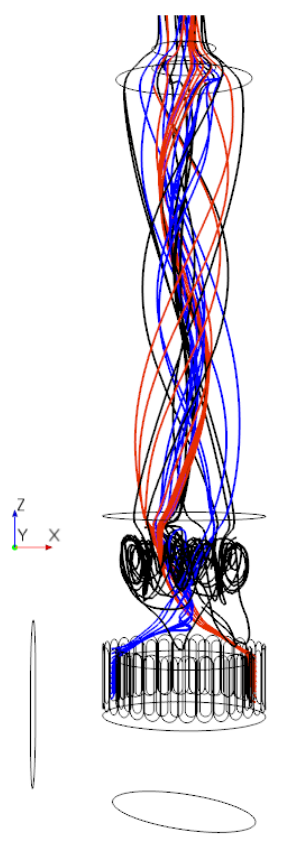

Figure 5: Streamlines at 150 CAD ATDC for URANS turbulence model, red) port 1, blue) port 2, black) vortex ring

In the first region, in the bottom of the cylinder near the surface of the piston, the exhaust gas is pulled down due to the downward motion of the piston. There is also flow separation and re-circulation near the piston surface. The second region with negative axial velocity is in the nearwall region. This region is observed in all of the presented CADs and is likely due to the separation and re-circulation of fresh air above the ports and near the cylinder wall. The third region, which is more obvious in 160 CAD ATDC, is due to the adverse pressure gradient in the center of the cylinder. This is because of the swirl motion which leads to a decrease in the axial velocity and re-circulation of flow. This central re-circulation region around the cylinder axis leads to a vortex breakdown.

By comparing the snapshots obtained using the URANS and LES models at $160 \mathrm{CAD}$, it can be observed that LES predicts a larger central area with negative axial velocity. Also, it can be seen that URANS cannot predict any central re-circulation region in the swirl core at 180 , 200 and 210 CAD ATDC, but LES predicts this central recirculation region. This is because of the URANS models incapability in the simulation of highly swirling flows [20]. It should be highlighted that there is no experimental results for this position for the full-size engine, but experimental results of a stationary model engine showed the central negative axial velocity for this region [4]. Furthermore, the velocity field for the LES model shows more fluctuations than URANS, as expected. In URANS, all fluctuations are averaged and the physics of the transient large-scale structure of turbulent fluctuation is lost. On the other hand in LES, a filtering function is applied such that large turbulence structures, in the form of eddies, are resolved and computed directly, while smaller eddies are modelled.

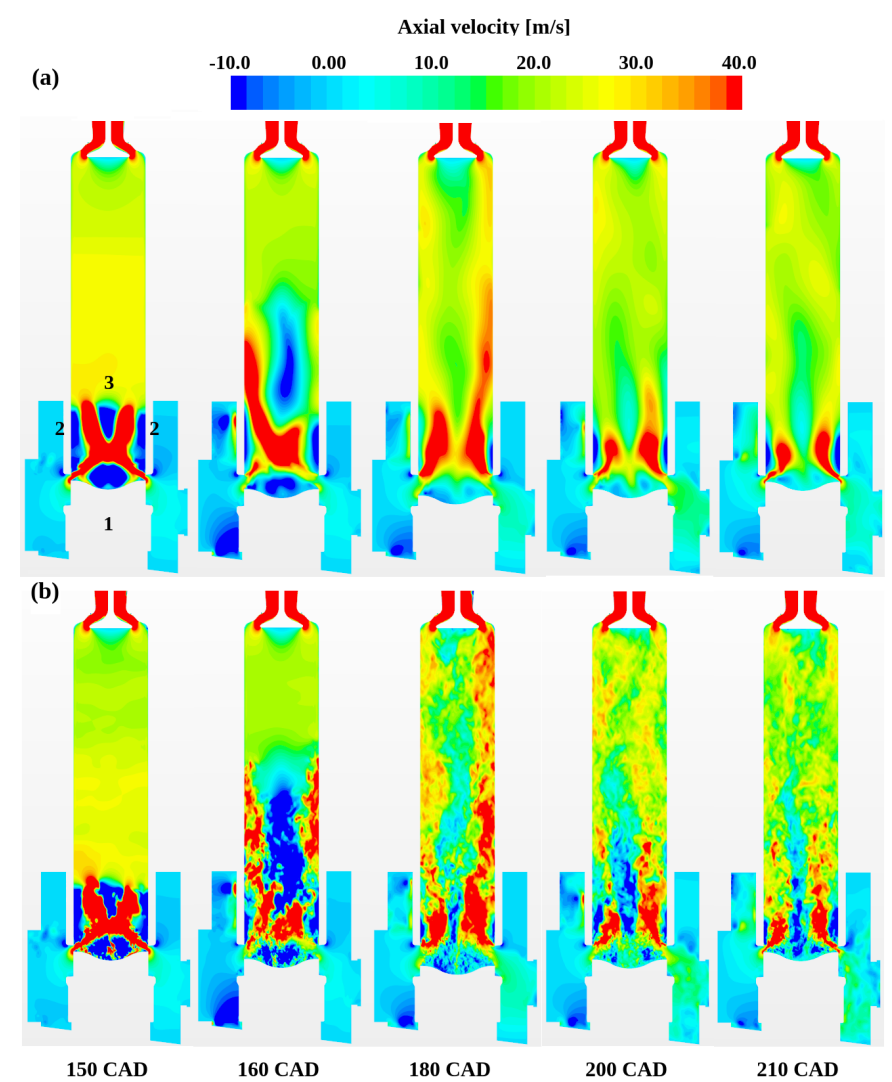

Figure 6: Distribution of the axial velocity at different CADs ATDC, (a) URANS, (b) LES

The distribution of in-cylinder tangential velocity is depicted in Figure 7 for the URANS and LES models. At the region near the scavenging ports, the tangential velocity increases from the center and towards the wall and then deceases near the cylinder wall. This tangential velocity profile matches a typical Burgers vortex profile which is composed of a forced vortex at the center and a free vortex around it. It is obvious that at 160 CAD ATDC, the high swirl flow has not reached the top of the cylinder, where the laser sheet is located. This clarifies the tangential ve- 
locity temporal trend in Figure $4 \mathrm{~b}$ which has a constant value in the range of 128-170 CAD ATDC. By comparison of the tangential velocity contours for the URANS and LES cases in Figure 7, it can be seen that LES predicts a more extended region of Burgers vortex in the upstream of the cylinder compared to the URANS (please see 200 and 210 CAD ATDC in Figure 7).

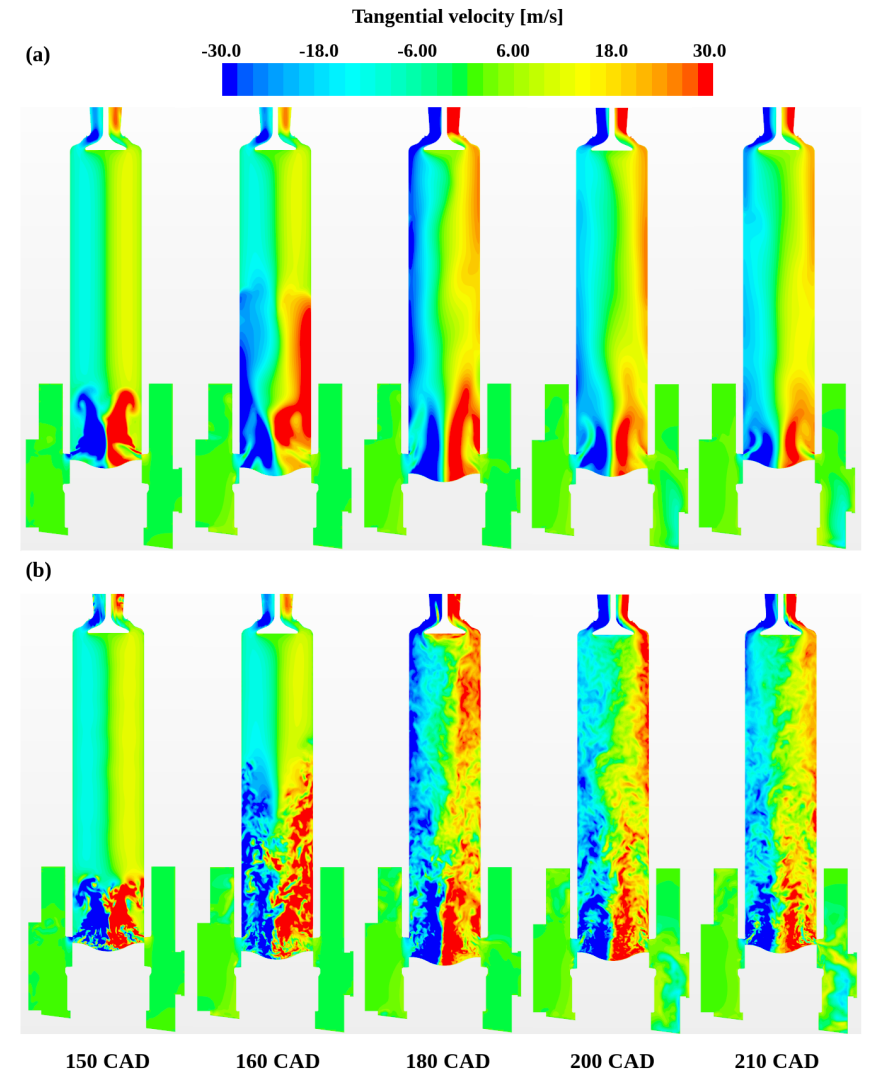

Figure 7: Distribution of the tangential velocity at different CADs ATDC, (a) URANS, (b) LES

It is also worth mentioning that URANS predicts a solid body rotation in the upstream of the cylinder. However, LES predicts a non-solid body rotation in this region. This is consistent with experimental results of the full-size engine [1] and the model engine [7] which indicate that the swirling flow inside the cylinder rotates as a non-solid body profile.

In this section, the radial distributions of the axial and tangential velocity components are presented and compared for the URANS and LES models at some important CADs and positions. The velocity components are sampled on some of the line probes which are clarified in Figure 1. The comparison of axial velocity profiles for the URANS and LES at 150, 180 and 210 CAD ATDC in the position of $z / D=1$ is presented in Figure 8 . The incoming flow reaches the position of $z / D=1$ at $150 \mathrm{CAD}$ ATDC and leads to a large variation in axial velocity. At this time, the LES and URANS models predict a similar profile, however, LES predicts a larger separation at the vicinity of those ports close to the intake port of the scav- enging box (the left side of Figure 8). The location of the intake port is shown in Figure 1. It is also worth mentioning that URANS cannot predict the negative axial velocity at 180 and 210 CADs ATDC and is incapable of predicting the central re-circulation zone at these CADs.

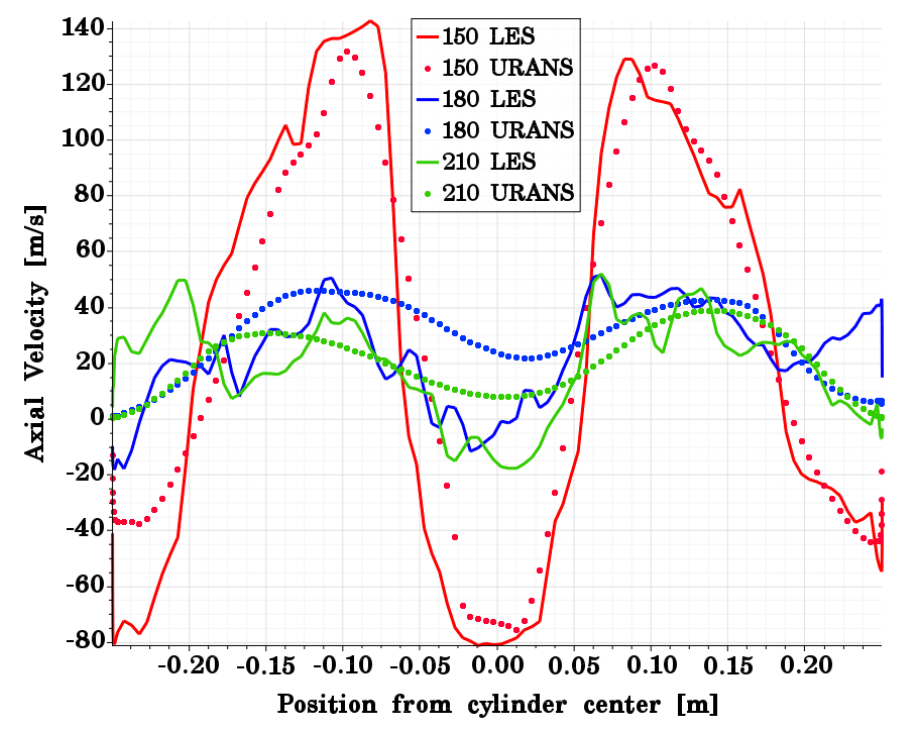

Figure 8: Comparison of axial velocity radial profiles at $z / D=1$, 150, 180 and 210 CAD ATDC for URANS and LES

Prediction of a correct tangential velocity and swirl is crucial in the vicinity of the fuel injectors in order to have a correct air-fuel mixing which has a significant effect on the combustion in these engines. Therefore, the radial profile of tangential velocity at the position of $z / D=4$ is presented in Figure 9 for 250 CAD ATDC. As it can be seen, the URANS model predicts a solid body rotation at this position. It is however observed that LES predicts a tangential velocity which is almost distributed uniformly at this position and this is consistent with experimental data [1, 21].

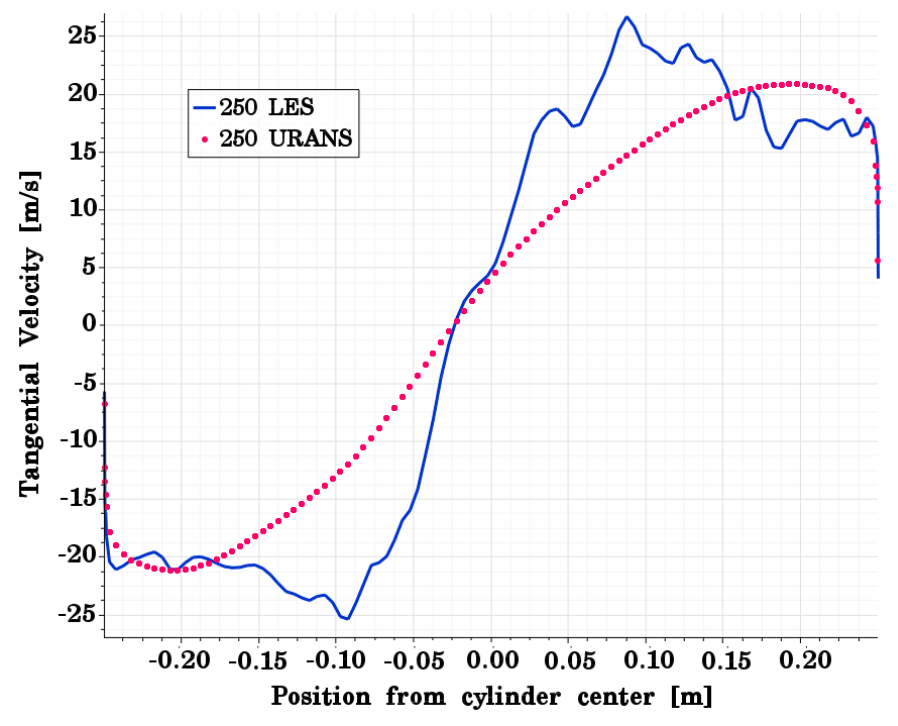

Figure 9: Comparison of tangential velocity radial profiles at $z / D=4,250$ CAD ATDC for URANS and LES 
To have more insight into the differences between the URANS and LES models in the simulation of swirling flow inside the marine engine, a comparison of the averaged angular momentum inside the cylinder divided by the incylinder charge mass is presented in Figure 10. After the opening of the scavenging ports, the angular momentum increases due to the inflow of the fresh scavenge air to the cylinder. The URANS and LES models almost predict a similar trend during the scavenging process. However, their predictions start to deviate after around 200 CAD ATDC. As it can be seen in Figure 9, the URANS and LES predict similar values of the tangential velocity for positions near the cylinder wall $(r \geq 0.18 \mathrm{~m})$. The main difference is in the prediction of the tangential velocity near the cylinder axis. LES predicts an almost uniform tangential velocity which leads to higher tangential velocity in the inner positions inside the cylinder in comparison with URANS that predicts a solid body rotation and a lower tangential velocity for positions close to the cylinder axis. This leads to a prediction of higher angular momentum by the LES model than the URANS model which can affect the air-fuel mixing and combustion process. This clarifies that the LES model performs better in capturing the qualitative velocity field inside the cylinder.

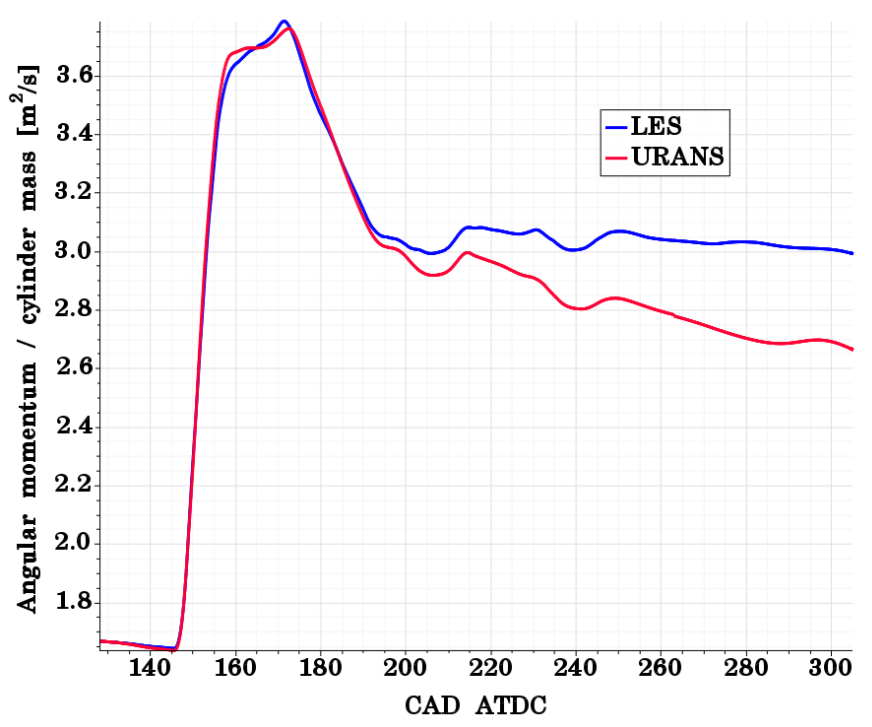

Figure 10: Comparison of cylinder averaged angular momentum divided by cylinder mass for URANS and LES

The iso-surfaces of pressure and axial velocity are presented and compared in Figure 11 to visualize the swirl centers at different CADs ATDC. The iso-surface of velocity presents the reversal region $\left(\mathrm{V}_{\mathrm{z}}=0\right)$. In the LES axial velocity iso-surfaces it is clear that the flow has a precessing vortex core [22] around the axis of the cylinder which is located at the boundary of positive and negative axial velocity. This is indicated by the blue iso-surface. It is observed that URANS cannot capture this phenomenon.

In order to evaluate the gas exchange during the scavenging process, a passive scalar is visualized for the URANS and LES at different CADs in Figure 12.

\section{URANS}

(a)
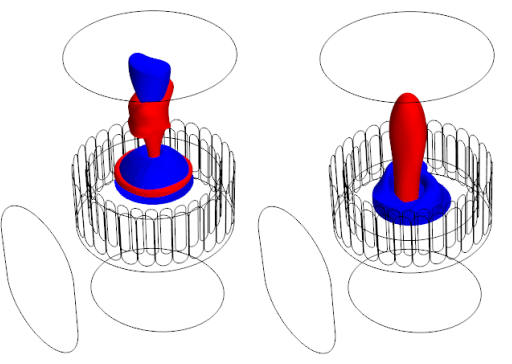

(b)

(c)

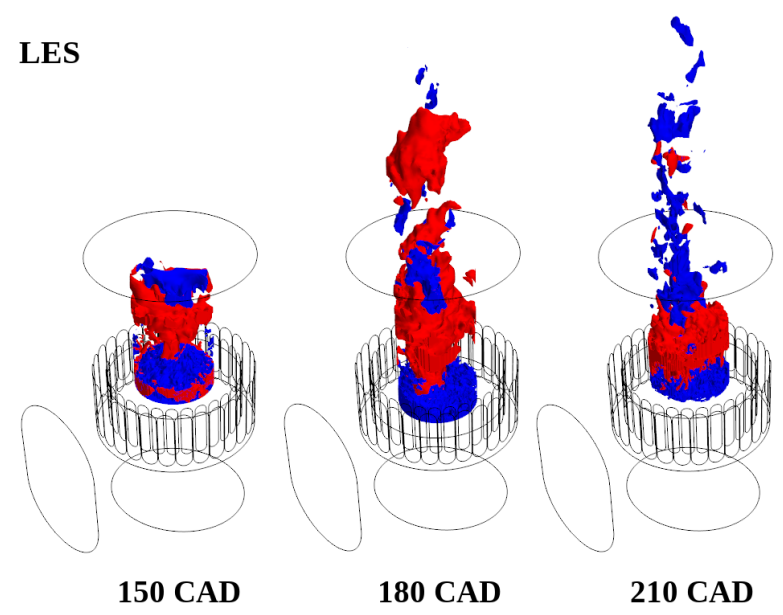

Figure 11: Iso-surfaces of axial velocity $=0$ (blue color) and pressure $=(a) 1.27$, (b) 1.46 and (c) 1.43 bar (red color)

The transport equation for the passive scalar does not affect the solution of other equations (including mass, momentum, and energy) and serves as an indication of the burned gas mass fraction. Hence regions with burned gas are indicated by red color while blue color indicates regions with charge air. As it can be seen, at 150 CAD ATDC the incoming air from the ports meet in the center of the cylinder and mix with the burned gas. At 165 CAD ATDC, it is obvious that mixing between air and burned gases is asymmetrical and irregular. The trapping of the burned gases in the center of the cylinder due to the swirling flow is obvious at 180 CAD ATDC, especially in the LES snapshot of the passive scalar. However, due to the efficient scavenging process in this engine, there is virtually no remaining burned gas inside the cylinder after EVC. The URANS and LES models predict a similar scavenging efficiency for the simulated engine (not shown).

The geometrical angle of the scavenging ports is one of the governing factors in the swirl strength of the incoming air through the scavenging ports. However, experimental studies [23] showed that the angle of the incoming flow direction does not match exactly the geometrical angle of the ports. In the current work, the effective flow angle is defined as the averaged angle between the cylinder radial direction and the effective velocity vector for the flow through the ports. The effective velocity vector for 
the flow through the ports is calculated from the surfaceaveraged tangential and radial components of the flow velocity through the ports on the interface between the cylinder and scavenging ports. The effective flow angle is presented in Figure 13 for the URANS and LES cases. As it can be seen, the effective flow angle after the opening of the scavenging ports is low and then starts to increase.

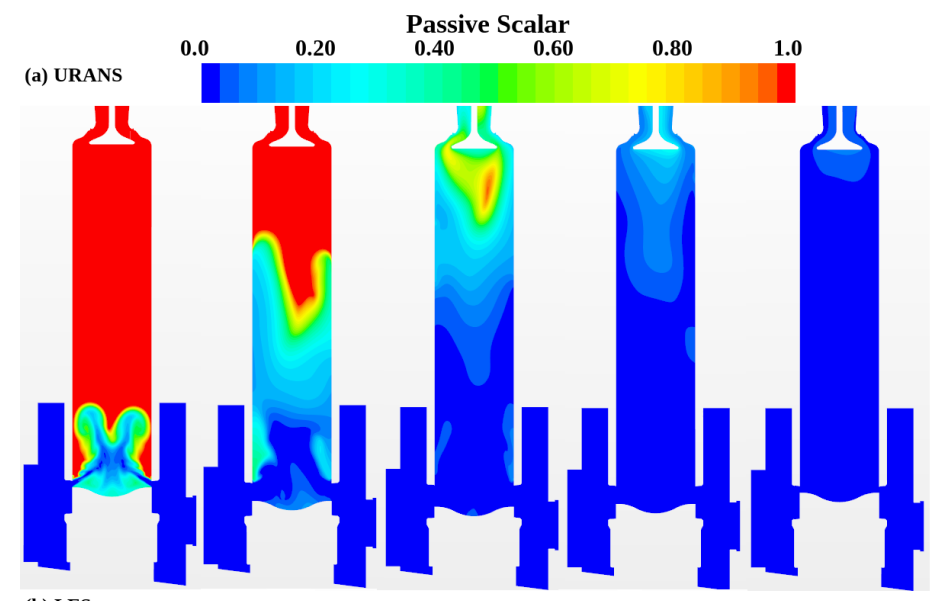

(b) LES

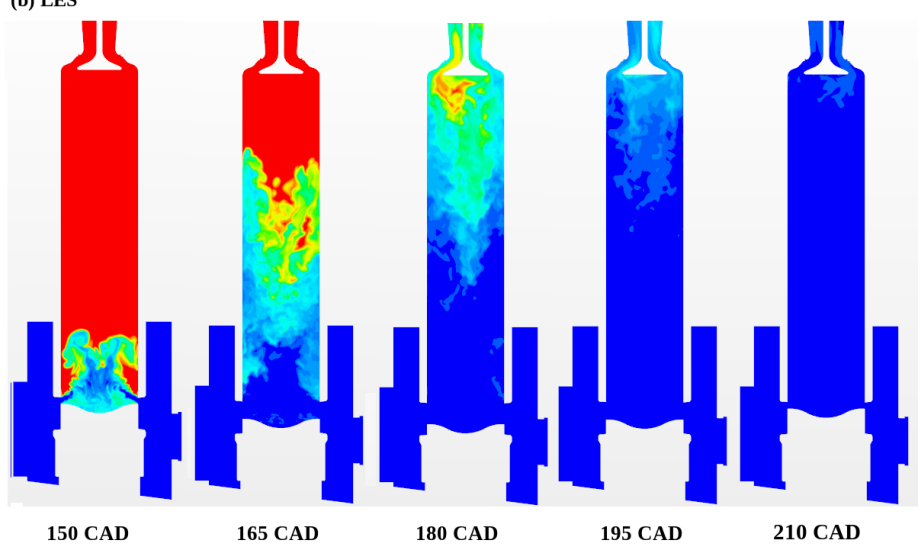

Figure 12: Distribution of burned gas inside the cylinder at different CADs for URANS and LES

This implies that the air enters into the cylinder mostly in the radial direction after the opening of the ports. This is due to the high flow rate of air through the small part of the ports which enforces the air in the radial direction. The effective flow angle increases until the swirl develops inside the cylinder. Around 157-160 CAD ATDC, the effective flow angle reaches $20^{\circ}$ which is the geometrical angle of the scavenging ports. The effective flow angle fluctuates around $20^{\circ}$ until 200 CAD ATDC and starts to decrease near the closing of the ports. The predicted effective flow angle for LES and URANS is more or less the same. However, the LES model shows a higher effective flow angle around 160 CAD ATDC. This is because the pressure at the inlet pressure boundary condition decreases around 147 CAD ATDC (please see Figure 2), and the radial velocity reduces accordingly. A similar trend can be observed at around 174 CAD ATDC (please see Figure 13).

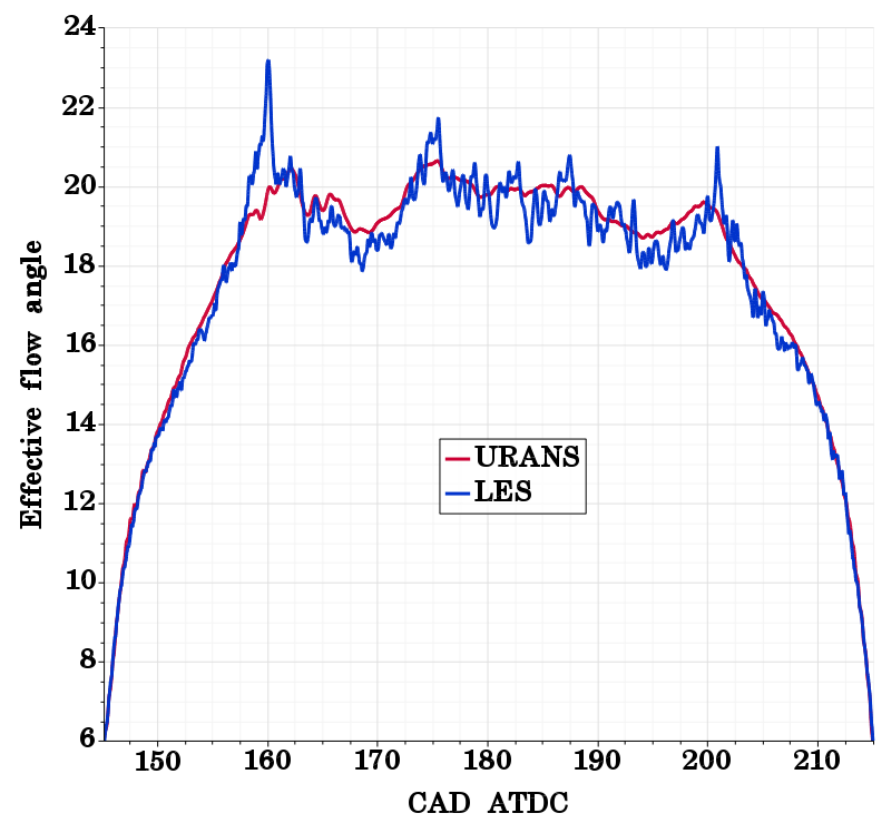

Figure 13: Comparison of the effective flow angle for URANS and LES during the scavenging process

\section{CONCLUSION}

A 3D-CFD simulation of the scavenging flow in a large two-stroke marine engine is presented and validated with experimental PIV data of axial and tangential in-cylinder velocities [1]. The URANS and LES turbulence models are utilized to simulate the in-cylinder flow during the scavenging process. The main conclusions are as follows:

1- There are three main regions of re-circulation zones in the engine. The central re-circulation region around the cylinder axis leads to a vortex breakdown. Based on the obtained results, URANS is incapable of predicting this region between the BDC and IPC while LES is able to capture this region.

2- LES has a better performance in the prediction of a Burgers vortex in the upstream of the cylinder near the scavenging ports. Furthermore, LES predicts a higher angular momentum inside the cylinder in comparison with URANS.

3- The accuracy of the tangential velocity and swirl in the top of cylinder where the fuel is injected is crucial to simulate the air-fuel mixing correctly. URANS predicts a solid body rotation for the tangential velocity in this region. However, LES predicts a non-solid body rotation (uniform profile) which is consistent with experimental results. Therefore, the LES performs better in capturing the qualitative velocity field inside the cylinder.

4- Analysis of the effective flow angle revealed that the flow angle does not match the geometrical angle of the ports in the beginning and end of the scavenging process due to higher radial velocity in these periods. 
5- The studied engine has an effective scavenging process at the simulated engine load and is able to replace the burned gas with fresh air almost completely. The URANS and LES models predict a similar scavenging efficiency.

\section{REFERENCES}

[1] J. Hult, S. Matlok, and S. Mayer, "Particle image velocimetry measurements of swirl and scavenging in a large marine two-stroke diesel engine," SAE Tech. Paper Ser., vol. 01-1173, 2014.

[2] K. M. Pang, N. Karvounis, J. H. Walther, and J. Schramm, "Numerical investigation of soot formation and oxidation processes under large twostroke marine diesel engine-like conditions using integrated CFD-chemical kinetics," Appl. Energy, vol. 169, pp. 874-887, 2016.

[3] C. S. Hemmingsen, K. M. Ingvorsen, S. Mayer, and J. H. Walther, "LES and RANS simulations of swirling flow in a dynamic model of a uniflow-scavenged cylinder," Int. J. Num. Meth. for Heat \& Fluid Flow, vol. 62, pp. 213-223, 2016.

[4] K. M. Ingvorsen, K. E. Meyer, J. H. Walther, and $\mathrm{S}$. Mayer, "Turbulent swirling flow in a dynamic model of a uniflow-scavenged two-stroke engine," Exp. Fluids, vol. 55, p. 1748, 2014.

[5] J. Hult and S. Mayer, "A methodology for laser diagnostics in large-bore marine two-stroke diesel engines," Meas. Sci. Technol., vol. 24, no. 4, pp. 1-10, 2013.

[6] S. Haider, T. Schnipper, A. Obeidat, K. E. Meyer, V. L. Okulov, S. Mayer, and J. H. Walther, "PIV study of the effect of piston position on the in-cylinder swirling flow during the scavenging process in large twostroke marine Diesel engines," J. Mar. Sci. Tech., vol. 18, pp. 133-143, 2013.

[7] K. M. Ingvorsen, K. E. Meyer, J. H. Walther, and S. Mayer, "Phase-locked stereoscopic PIV measurements of the turbulent swirling flow in a dynamic model of a uniflow-scavenged two-stroke engine cylinder," in 10th international symposium on particle image velocimetry, (Delft, The Netherlands), July 2013.

[8] N. W. Sung and D. J. Patterson, "Air motion in a two stroke engine cylinder - the effects of exhaust geometry," SAE Trans., pp. 2534-2544, 1982. Paper No. 820751.

[9] H. Nakagawa, S. Kato, M. Tateishi, T. Adachi, H. Tsujimura, and M. Nakashima, "Airflow in the cylinder of a 2-stoke cycle uniflow scavenging diesel engine during compression stroke," Jpn. Soc. Mech. Eng., vol. 33, no. 3, pp. 591-598, 1990.
[10] S. S. Goldsborough and P. V. Blarigan, "Optimizing the scavenging system for a two-stroke cycle, free piston engine for high efficiency and low emissions: A computational approach," SAE Tech. Paper Ser., vol. 1, pp. 1-22, 2003.

[11] A. Obeidat, S. Haider, K. E. Meyer, T. Schnipper, S. Mayer, and J. H. Walther, "Influence of piston position on the scavenging and swirling flow in two-stroke diesel engines," in EUROMECH Colloquium 525, Instabilities and transition in three-dimensional flows with rotation, (Lyon-Ecully, France), pp. -, June 2011.

[12] A. Obeidat, T. Schnipper, K. M. Ingvorsen, S. Haider, K. E. Meyer, S. Mayer, and J. H. Walther, "Large eddy simulations of the influence of piston position on the swirling flow in a model two-stroke diesel engine," Int. J. Num. Meth. for Heat \& Fluid Flow, vol. 24, no. 2, pp. 325-341, 2014.

[13] L. Cui, T. Wang, K. Sun, Z. Lu, Z. Che, and Y. Sun, "Numerical analysis of the steady-state scavenging flow characteristics of a two-stroke marine engine," SAE Technical Paper, vol. 01-0558, 2017.

[14] M. I. Lamas and C. G. R. Vidal, "Computational fluid dynamics analysis of the scavenging process in the MAN B\&W 7S50MC two-stroke marine Diesel engine," J. Ship. Res., vol. 56, no. 3, pp. 154-161, 2012.

[15] F. Andersen, J. Hult, K. Nogenmyr, and S. Mayer, "Numerical investigation of the scavenging process in marine two-stroke diesel engines," in SAE/KSAE 2013 International Powertrains, Fuels \& Lubricants Meeting, (Seol, South Korea), pp. Paper No. 201301-2647, SAE, October 2013.

[16] Z. Cao, T. Wang, K. Sun, Z. Lu, L. Cui, and Y. Gui, "Numerical analysis of scavenging process in a large marine two-stroke diesel engine," SAE Technical Paper, vol. 01-2201, 2017.

[17] M. I. Foteinos, A. Papazoglou, N. P. Kyrtatos, A. Stamatelos, O. Zogou, and A.-M. Stamatellou, "A threezone scavenging model for large two-stroke uniflow marine engines using results from CFD scavenging simulations," Energies, vol. 12, no. 9, p. 1719, 2019.

[18] K. M. Ingvorsen, K. E. Meyer, T. Schnipper, J. H. Walther, and S. Mayer, "Swirling flow in model of large two-stroke diesel engine," in 16th Int. Symp. on Applications of Laser Techniques to Fluid Mechanics, (Lisbon, Portugal), July 2012.

[19] T.-H. Shih, W. W. Liou, A. Shabbir, Z. Yang, and $J$. Zhu, "A new $k-\epsilon$ eddy viscosity model for high Reynolds number turbulent flows," Computers \& Fluids, vol. 24, no. 3, pp. 227-238, 1995.

[20] A. J. Hoekstra, J. J. Derksen, and H. E. A. Van Den Akker, "An experimental and numerical study of turbulent swirling flow in gas cyclones," Chem. Eng. Sci., vol. 54, pp. 2055-2065, 1999. 
[21] K. M. Ingvorsen, K. E. Meyer, J. H. Walther, and S. Mayer, "PIV and LDA measurements of the swirling flow in a low-speed two-stroke diesel engine," in 10th European Fluid Mechanics Conference (J. N. Sørensen, ed.), (Copenhagen, Denmark), EUROMECH, September 2014.

[22] S. V. Alekseenko, P. A. Koubin, V. L. Okulov, and S. I. Shtork, "Helical vortices in swirl flow," J. Fluid Mech., vol. 382, pp. 195-243, 1999.

[23] W. Rizk, "Experimental studies of mixing processes and flow configurations in two-cycle engine scavenging," Proc. Inst. Mech. Eng., vol. 172, no. 10, pp. 417427, 1958.

\section{ACKNOWLEDGMENT}

The authors gratefully acknowledge the financial support from the Independent Research Fund Denmark (DFF) and MAN Energy Solutions under the grant number $8022-$ 00143B. The authors also would like to thank MAN Energy Solutions, Denmark for sharing the experimental data. The computation was performed using the Niflheim cluster at the Technical University of Denmark (DTU).

\section{CONTACT INFORMATION}

Arash Nemati,

Ph.D. student, Technical University of Denmark, Department of Mechanical Engineering

Web: www.mek.dtu.dk

E-mail: arnem@mek.dtu.dk,

E-mail: nemati.arash.mech@gmail.com 